\title{
A relative Lubin-Tate theorem via higher formal geometry
}

\author{
AARON MAZEL-GeE \\ ERIC PETERSON \\ NATHANIEL STAPLETON
}

\begin{abstract}
We formulate a theory of punctured affine formal schemes, suitable for describing certain phenomena within algebraic topology. As a proof-of-concept we show that the Morava $K$-theoretic localizations of Morava $E$-theory, which arise in transchromatic homotopy theory, corepresent a Lubin-Tate-type moduli problem in this framework.
\end{abstract}

$14 \mathrm{~L} 05 ; 55 \mathrm{~N} 22$

\section{Introduction}

Associated to a fixed formal group law $\Gamma$ of finite height $h$ over the field $k=\mathbb{F}_{p^{h}}$, there is a cohomology theory $E_{h}$ called Morava $E$-theory. Its coefficient ring can be noncanonically identified as

$$
E_{h}^{0} \cong \mathbb{W}_{k} \llbracket u_{1}, \ldots, u_{h-1} \rrbracket .
$$

Both $E$-theory and this ring are intimately connected to the infinitesimal deformation theory of formal groups: the associated formal scheme $\mathrm{LT}_{h}=\operatorname{Spf} E_{h}^{0}$ is known as Lubin-Tate space, which classifies deformations of $\Gamma$ [15]. More precisely, when $R$ is a complete local ring with residue field $k$, maps $\operatorname{Spf} R \rightarrow \mathrm{LT}_{h}$ are in bijective correspondence with $\star$-isomorphism classes of formal group laws over $R$ which reduce over the special fiber to the specified formal group law $\Gamma$. Given a complex orientation $\operatorname{Spf} E_{h}^{0} \mathbb{C} \mathrm{P}^{\infty} \cong \operatorname{Spf} E_{h}^{0} \llbracket x \rrbracket$ of $E$-theory, the resulting formal group law associated to the formal group

$$
\operatorname{Spf} E_{h}^{0} \mathbb{C P}^{\infty} \rightarrow \operatorname{Spf} E_{h}^{0}=\mathrm{LT}_{h}
$$

is an example of such a versal formal group law; that is, the indicated bijection is induced by pulling back this formal group law along maps $\operatorname{Spf} R \rightarrow \mathrm{LT}_{h}$.

Transchromatic homotopy theory studies phenomena related to the self-interaction of the height filtration of the moduli of formal groups as appearing in chromatic homotopy theory. One object of interest in this subfield is $L_{K\left(h^{\prime}\right)} E_{h}$, the Bousfield localization at $K\left(h^{\prime}\right)$ of Morava $E$-theory, where the Morava $K$-theory $K\left(h^{\prime}\right)$ is the reduction 
of $E_{h^{\prime}}$ to the special fiber of $\mathrm{LT}_{h^{\prime}}$. For $h^{\prime}>h$ the spectrum $L_{K\left(h^{\prime}\right)} E_{h}$ is zero, and for $0 \leq h^{\prime} \leq h$ it follows from a formula of Mark Hovey [7, Lemma 2.3] that the coefficient ring of $L_{K\left(h^{\prime}\right)} E_{h}$ can be expressed as

$$
\left(L_{K\left(h^{\prime}\right)} E_{h}\right)^{0} \cong \mathbb{W}_{\mathbb{F}_{p^{h}}} \llbracket u_{1}, \ldots, u_{h-1} \rrbracket\left[u_{h^{\prime}}^{-1}\right]_{I_{h^{\prime}}}^{\wedge},
$$

where $I_{h^{\prime}}$ denotes the ideal $I_{h^{\prime}}=\left(p, u_{1}, \ldots, u_{h^{\prime}-1}\right)$. Haynes Miller has suggested that this ring should be similarly studied through the moduli problem it represents; but from the point of view of formal geometry, this ring is extremely unfriendly. Here is an inexhaustive list of complaints.

- It is not a complete local ring, so cannot give rise to a formal scheme in the most naive, 1970s sense of the phrase that has served an enormous amount of algebraic topology perfectly well.

- Inverting topologically nilpotent elements destroys adic topologies. The adic topology on $k \llbracket x \rrbracket$ comes from declaring linear translates of powers of the ideal $(x)$ to be a basis of open sets. In turn, this has the effect of requiring that a continuous map $k \llbracket x \rrbracket \rightarrow R$ to a discrete ring $R$ sends $x$ to a nilpotent element, as continuous maps respect the ideals of definition. There are therefore no maps $k((x)) \rightarrow R$ restricting to continuous maps $k \llbracket x \rrbracket \rightarrow k((x)) \rightarrow R$, which is highly unsatisfactory when identifying schemes with their functors of points.

- It is generally only complete against some of the power series generators. So, even making use of the available completeness, when $h^{\prime}<h-1$ there remain power series generators for which the ring is not complete. Power series rings without their completion taken into account are quite intimidating.

Thus, the classical framework of formal geometry fails to account for the subtleties of this ring.

Nevertheless, rings of this sort have been gaining importance in chromatic homotopy theory. Here is a small sampling of applications.

- Takeshi Torii has worked extensively with $L_{K(h-1)} E_{h}$ (ie in the case $h^{\prime}=$ $h-1)$, producing many interesting results about transchromatic phenomena and interrelationships among the stabilizer groups $[24 ; 25 ; 26 ; 27 ; 28]$.

- Tyler Lawson and Niko Naumann have encountered these kinds of rings while studying the interplay of the $K(1)$ - and $K(2)$-local obstructions to realizing $\mathrm{BP}\langle 2\rangle$ as an $E_{\infty}$-ring spectrum [14]. 
- Such rings appear in and around the Tate construction, especially in connection to results about the pro-spectrum $\mathbb{C P}_{-\infty}^{\infty}$ arising from stunted projective space. For example, this powers work of Matthew Ando, Jack Morava and Hal Sadofsky on a phenomenon known as "blueshift" $[2 ; 3]$.

- Meromorphic formal geometry and formal Laurent series rings appear in the "sharp construction" of Matthew Ando, Christopher French and Nora Ganter, which connects $\mathbb{C P}_{-\infty}^{\infty}$ to the study of $\mathrm{MU}\langle 2 k\rangle$-orientations [1].

- John Greenlees and Neil Strickland have studied a form of transchromatic character theory involving the ring $E_{h}^{0}\left[u_{h^{\prime}}^{-1}\right] / I_{h^{\prime}}$, which corresponds to the functions on the punctured neighborhood $X_{h^{\prime}} \backslash X_{h^{\prime}-1}$ for a filtration $X_{*}$ on Lubin-Tate space [6].

- The third author has used the spectrum $L_{K\left(h^{\prime}\right)} E_{h}$ and the $p$-divisible group $\left(\operatorname{Spf} E_{h}^{0} \mathbb{C P}^{\infty}\right)\left[p^{\infty}\right] \otimes L_{K\left(h^{\prime}\right)} E_{h}^{0}$ to develop a variety of results concerning transchromatic character theory and group-cohomological data; see Schlank and the third author [21] and the third author [22;23].

It is therefore important to sort out what variation on formal geometry houses these objects, given the past successes of formal geometry at organizing and interpreting results in algebraic topology.

Our goal is to propose a framework in which the ring $L_{K\left(h^{\prime}\right)} E_{h}^{0}$ and various natural maps concerning it can be studied. Kazuya Kato has already ventured in this direction [12] as part of a program to understand "higher local fields", the relevant higher-dimensional analogues of local function fields of smooth points on algebraic curves. Other workers in this same area, for instance, Kazuya Kato and Shuji Saito [13], Shuji Saito [19; 20], Denis Osipov [16, Section 3; 17, Sections 2.2 and 3.2], and generally those trying to understand the higher adèlic program initiated by Beĭlinson and Parshin (see Beĭlinson [5] and Huber [8, Section 3.2]) grapple with many of the phenomena we encounter. What we contribute lies atop their substantial groundwork. Geometrically, the broad idea is to study analytic functions on deleted neighborhoods within formal schemes. This necessitates not just working with a localization of a complete ring, but also remembering the complete ring from which it stems, together with both of their defining direct and inverse systems. In effect, this endows various elements of the ring with distinct "rates of convergence". Along with more minor examples, we prove a Lubin-Tate result for $L_{K\left(h^{\prime}\right)} E_{h}^{0}$ in this extended setting:

Main theorem (Proven below as Theorem 36) Fix a finite ground field $k$ of positive characteristic $p$ and a formal group law $\Gamma$ of finite height $h$ over $k$. Select an infinitesimal deformation $X$ of Spec $k$ and a "punctured affine formal scheme" $X^{\prime}=$ 
Spp $R$ over $X$. Then, for each $h^{\prime} \leq h$, there is an equivalence between commuting diagrams of the shape

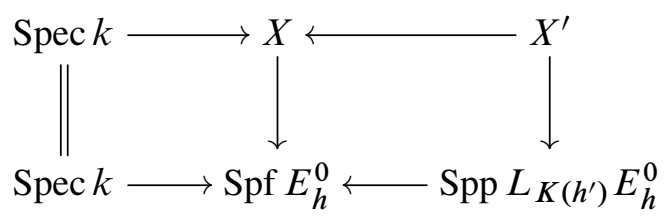

and formal group laws $\mathbb{G}$ over $X$ deforming $\Gamma$ with base change to $X^{\prime}$ of height $h^{\prime}$.

Acknowledgements There are a number of people to thank for their contributions to this paper: Olga Stroilova contributed substantially to the initial ideas; Dustin Clausen very helpfully suggested that we look to the algebraic geometry of local fields of large dimension for guidance; Jared Weinstein gave us a useful guided tour of many existing extensions of the theory of formal schemes; Charles Rezk is responsible for all three of our interests in the cohomology theory $L_{K\left(h^{\prime}\right)} E_{h}$; Neil Strickland has been persistently inspiring in his use of algebraic geometry as an organizing principle in algebraic topology; Adam Prescott suggested the name "pipe rings" over a multitude of worse ideas we had previously tried; Tobias Barthel contributed to a variety of helpful conversations; Justin Noel read a draft of this paper and caught a number of crucial errors; Geoffrey Powell also read an early version and provided very helpful structural remarks; an anonymous referee contributed a number of comments that led to significant clarifications and improvements; and the generosities of Haynes Miller, Peter Teichner and Constantin Teleman all came together to make possible the visits of the first two authors to MIT, where all the work on this paper took place. The first author was supported in part by NSF GRFP grant DGE-1106400; the first and second authors were both supported in part by UC Berkeley's geometry and topology RTG grant, which is part of DMS-0838703; and the third author was partially supported by NSF grant DMS-0943787. Finally, all three authors thoroughly enjoyed the comfort and hospitality of the Muddy Charles.

\section{Continuity and higher formal geometry following Kato}

The goal of this section is to construct and study a category of "pipe rings" satisfying the following desiderata.

(1) The usual category of profinite rings and continuous maps contributes a full subcategory of pipe rings. That is to say: pipe rings will carry a "generalized topology", which reduces to the adic topology in that full subcategory. 
(2) The localization morphism $\pi_{0} E_{h} \rightarrow \pi_{0} L_{K\left(h^{\prime}\right)} E_{h}$ belongs to this category, as do the more general iterated localization morphisms

$$
\pi_{0} E_{h} \rightarrow \pi_{0} L_{K\left(h^{\prime}\right)} E_{h} \rightarrow \pi_{0} L_{K\left(h^{\prime \prime}\right)} L_{K\left(h^{\prime}\right)} E_{h} \rightarrow \cdots
$$

Kato has constructed such a category of rings with these properties in an effort to understand the continuity properties of higher local fields [12]. Using our terminology, we recall his construction and point out various properties and constructions which we now need but which he has not already recorded.

Definition 1 Let Pipes -1 denote the category of finite sets and Pipes ${ }_{0}$ the category of profinite sets, which we refer to as (-1)-pipes and 0-pipes respectively. For $n \geq 1$, we inductively define the category Pipes $_{n}$ of $n$-pipes by the formula

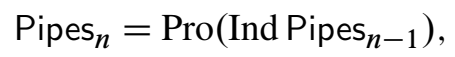

and we refer to $n$ as the length. (Note that there is an exception in the bottom case: Pipes $_{0}$ is not equivalent to Pro(Ind Pipes-1).) There are inclusions Pipes ${ }_{n-1} \rightarrow$ Pipes $_{n}$ given by sending an object of Pipes ${ }_{n-1}$ to its associated constant system in Pro(Ind Pipes $\left.{ }_{n-1}\right)=$ Pipes $_{n}$, and these inclusions are fully faithful and preserve finite products. We denote the sequential colimit along these inclusions by $\mathrm{Pipes}_{\infty}$, and refer to its objects simply as pipes. ${ }^{1}$

Remark 2 The above definition is a superficial variation on Kato's categories $\mathcal{F}_{n}$ (see [12, page 166]); namely, $\mathcal{F}_{n}=$ Ind Pipes $_{n-1}$ for $n>0$. This difference is informed by our respective applications: we are interested in complete rings, where it is useful to consider pro-objects, and Kato is interested in fraction fields of rings, where it is useful to consider ind-objects.

Definition 3 The category Pipes $_{n}$ admits finite products (see Isaksen [9, Proposition 11.1]), so we may speak of its associated category of (commutative, unital) ring objects PipeRings $_{n}=$ Rings $\left(\right.$ Pipes $\left._{n}\right)$. As shorthand, we refer to an object of PipeRings ${ }_{n}$ as an $n$-pipe ring. The inclusions Pipes $_{n-1} \rightarrow$ Pipes $_{n}$ preserve finite products and so induce inclusions of categories of ring objects. In the aggregate these form the category PipeRings $\infty$ of pipe rings.

${ }^{1}$ The word "pipe" here is an acronym for Pro-Ind-Pro Ensemble. 


\subsection{An embedding condition}

To simplify notation, in this subsection we will write Hom-sets as $[-,-]$, regardless of the ambient category.

Definition 4 The constant system at a singleton set gives a terminal object $1 \in$ Pipes $_{\infty}$, and we define a functor Pipes $_{\infty} \rightarrow$ Sets by

$$
S \mapsto[1, S]=: \underline{S},
$$

called (set-theoretic) realization.

Cautionary remark 5 This notation is "opposite" that of Kato. He writes $\underline{S}$ for a pipe lifting a set $S$, whereas we take the pipe to be the more fundamental object and so prefer to use an unadorned symbol to denote it.

Remark 6 This definition can also be given inductively. On the one hand, if $S \in \mathrm{Pipes}_{n}$ is given as a pro-system $\left\{S_{\alpha}\right\}_{\alpha}$, then (considering each $S_{\alpha} \in$ Pipes $_{n}$ ) we have that

$$
\underline{S}=[1, S]=\left[1, \lim _{\alpha} S_{\alpha}\right]=\lim _{\alpha}\left[1, S_{\alpha}\right]=\lim _{\alpha} \underline{S_{\alpha}} .
$$

On the other hand, if each $S_{\alpha}$ is given as an ind-system $\left\{\left(S_{\alpha}\right)_{\beta}\right\}_{\beta}$ of $(n-1)$-pipes, then we analogously have

$$
\underline{S_{\alpha}}=\left[1, S_{\alpha}\right]=\left[1, \underset{\beta}{\operatorname{colim}}\left(S_{\alpha}\right)_{\beta}\right]=\underset{\beta}{\operatorname{colim}}\left[1,\left(S_{\alpha}\right)_{\beta}\right]=\underset{\beta}{\operatorname{colim}} \underline{\left(S_{\alpha}\right)_{\beta}}
$$

since 1 , as a finite set, is a small object. Thus, taking the realization is exactly the iterative process of taking limits of pro-systems and colimits of ind-systems. This implies that realization commutes with finite limits and in particular with finite products, so it induces a functor PipeRings $s_{\infty} \rightarrow$ Rings.

This functor should be thought of as "forgetful", in the sense of sending a topologized ring to its underlying ring. As one expects, this functor is not injective on objects; just as a given set can support many topologies, so do sets support many pipe structures. However, as we have set up the category Pipes $_{\infty}$, there is no reason to even expect realization to be faithful. This is a more serious problem.

Definition 7 [12, Definition 1] To control this aspect of realization, we make two further inductive definitions.

(a) Every (-1)-pipe and 0 -pipe is called fine. An $n$-pipe $Y$ is called fine if it can be expressed as a pro-system $\left\{Y_{\alpha}\right\}_{\alpha}$ of ind-systems $\left\{\left(Y_{\alpha}\right)_{\beta}\right\}_{\beta}$ such that:

- Each $\left(Y_{\alpha}\right)_{\beta}$ is a fine $(n-1)$-pipe.

- The induced map $\left(Y_{\alpha}\right)_{\beta} \rightarrow \underline{Y_{\alpha}}$ is injective for every choice of $\alpha$ and $\beta$. 
(b) Every (-1)-pipe is called cofine. A 0 -pipe $X$ is called cofine if it can be expressed as a pro-system $\left\{X_{\lambda}\right\}_{\lambda}$ of finite sets such that the induced map $\underline{X} \rightarrow X_{\lambda}$ is surjective for each choice of $\lambda$. Generally, an $n$-pipe $X$ is called cofine if it can be expressed as an ind-system $\left\{X_{\lambda}\right\}_{\lambda}$ of pro-systems $\left\{\left(X_{\lambda}\right)_{\mu}\right\}_{\mu}$ such that:

- Each $\left(X_{\lambda}\right)_{\mu}$ is a cofine $(n-1)$-pipe.

- The induced map $\underline{X} \rightarrow \underline{X_{\lambda}}$ is surjective for every choice of $\lambda$.

Lemma 8 The properties of being fine and cofine are both preserved by the inclusions Pipes $_{n-1} \rightarrow$ Pipes $_{n}$.

Altogether, the point is the following lemma:

Lemma 9 [12, Lemma 1.2] The realization functor is faithful whenever the source is cofine and the target is fine.

Proof We consider the map $\varphi_{X, Y}:[X, Y] \rightarrow[\underline{X}, \underline{Y}]$ induced by the realization functor, where $X$ is cofine and $Y$ is fine. There exists an integer $n$ for which both $X$ and $Y$ are realized as $n$-pipes and all maps $X \rightarrow Y$ are exhibited as maps of $n$-pipes. It thus suffices to check the situation where the source and target have the same length.

The statement is clear for $(-1)-$ and 0 -pipes. So, suppose that the statement holds for $(n-1)$-pipes. We then show that $\varphi_{X, Y}$ is injective for $X$ cofine and $Y$ fine as follows:

$$
\begin{aligned}
& {[X, Y]=\lim _{\alpha} \operatorname{colim}\left[X_{\lambda}, Y_{\alpha}\right]} \\
& =\lim _{\alpha} \operatorname{colim}_{\lambda} \lim _{\nu} \operatorname{colim}_{\beta}\left[\left(X_{\lambda}\right)_{\nu},\left(Y_{\alpha}\right)_{\beta}\right] \\
& \subset \lim _{\alpha} \operatorname{colim} \lim _{\nu} \operatorname{colim}_{\beta}\left[\underline{\left(X_{\lambda}\right)_{\nu}}, \underline{\left(Y_{\alpha}\right)_{\beta}}\right] \quad \text { (inductive assumption) } \\
& \subset \lim _{\alpha} \operatorname{colim} \lim _{\nu}\left[\left(X_{\lambda}\right)_{\nu}, \underline{Y_{\alpha}}\right] \\
& =\lim _{\alpha} \operatorname{colim}\left[\operatorname{colim}\left(X_{\lambda}\right)_{\nu}, \underline{Y_{\alpha}}\right] \\
& =\lim _{\alpha} \operatorname{colim}\left[\underline{X_{\lambda}}, \underline{Y_{\alpha}}\right] \\
& \subset \lim _{\alpha}\left[\underline{X}, \underline{Y_{\alpha}}\right] \\
& =\left[\underline{X}, \lim _{\alpha} \underline{Y_{\alpha}}\right] \\
& =[\underline{X}, \underline{Y}] \text {. }
\end{aligned}
$$

This completes the proof. 
This lemma demonstrates that PipeRings ${ }_{\infty}$ does indeed satisfy our first desideratum. More to the point, it illustrates the analogy with the classical situation of topologized sets, and indicates that we can indeed consider an $n$-pipe as determining a "generalized topology" on its set-theoretic realization.

These conditions are reminiscent of an analogous situation in abstract homotopy theory, where a model structure on a category determines which are the "right" objects to map to and from. Unfortunately, despite substantial effort, we have been unable to precisely pin down this analogy. Instead, let us record the following conjecture:

Conjecture 10 (Pipe dream) For every pipe $X$, there exists an initial cofine pipe $X^{c}$ over $X$ such that the map $X^{c} \rightarrow X$ induces an isomorphism $\underline{X^{c}} \rightarrow \underline{X}$. Dually, for every pipe $Y$, there exists a terminal fine pipe $Y^{f}$ under $Y$ such that the map $Y \rightarrow Y^{f}$ induces an isomorphism $\underline{Y} \rightarrow \underline{Y f}$. Finally, there is a class of weak equivalences $W$ for which these compute the derived maps:

$$
\operatorname{Hom}_{\text {Pipes }_{\infty}\left[W^{-1}\right]}(X, Y) \cong \operatorname{Hom}_{\text {Pipes }_{\infty}}\left(X^{c}, Y^{f}\right) .
$$

Remark 11 A cofineification functor for $\mathrm{Pipes}_{0}$ can be constructed as follows. Let $X$ be a profinite set, presented as a pro-system $\left\{X_{\alpha}\right\}_{\alpha}$ of finite sets. Each finite set $X_{\alpha}$ in the pro-system has a subset $X_{\alpha}^{\prime}$ of elements that persist in the inverse limit: these are precisely the elements which are in the image of $X_{\beta} \rightarrow X_{\alpha}$ for all $\beta<\alpha$. These assemble into a sub-pro-system $X^{\prime} \hookrightarrow X$ (indexed on the same diagram), which is clearly the desired cofineification and is clearly unique up to unique isomorphism.

This suggests the following more general construction. If $X$ is an $n$-pipe, presented as a pro-system $\left\{X_{\alpha}\right\}_{\alpha} \in$ Ind Pipes $_{n-1}$, then by fixing any particular $X_{\alpha}$ in the system we can consider the objects $X_{\beta} \rightarrow X_{\alpha}$ over it, each of which has a corresponding image factorization

$$
X_{\beta} \rightarrow \operatorname{im}\left(X_{\beta} \rightarrow X_{\alpha}\right) \hookrightarrow X_{\alpha} .
$$

For $\gamma<\beta$, then there is a canonical monomorphism $\operatorname{im}\left(X_{\gamma} \rightarrow X_{\alpha}\right) \rightarrow \operatorname{im}\left(X_{\beta} \rightarrow X_{\alpha}\right)$, and altogether this gives a pro-system of images over $X_{\alpha}$. However, unlike in the case where $X_{\alpha}$ was a finite set, we cannot guarantee that the pro-system of images can be replaced by a constant system, and it seems that the core feat of any construction of a cofineification functor on $n$-pipes would be to work around this fact. Some further discussion of this and related issues can be found in Appendix A.

\subsection{Closed ideals}

In algebraic geometry, one studies rings through their associated categories of modules and, in particular, through their ideals From a categorical perspective, ideals should be 
thought of as kernels of ring maps; then, just as the restriction of continuity on maps of topologized rings gives rise to the notion of a closed ideal, so should our "generalized topologies" determine the correct notion of an ideal in our setting. In this section, we make this notion precise and study some of its basic features, although we explore little beyond what we will need in the remainder of the paper. The interested reader is encouraged to refer to Appendix B for a more thorough exploration of these generalized topologies in terms of the spectrum of a pipe ring. Throughout, we will make quiet use of basic facts about pro-categories, excellent references for which are Isaksen's papers $[9 ; 10]$.

Definition 12 Let $R$ be a pipe ring. An ideal of $R$ is a (categorical) monomorphism $I \rightarrow R$ of $R$-modules exhibiting $I$ as the kernel of some pipe ring map $R \rightarrow R / I$ inducing a surjection $\underline{R} \rightarrow \underline{R} / \underline{I}$ of rings under set-theoretic realization. An ideal $\underline{I} \subset \underline{R}$ of the set-theoretic realization is said to be closed when it is the image under realization of some ideal $I$ of $R$ as a pipe ring.

Central to basic algebraic geometry are the notions of being complete and of being local. We now give analogous definitions in our setting.

Definition 13 Restricting the iterated action map $R^{\times(n-1)} \times I \rightarrow I$ to $I^{\times(n-1)} \times I$ gives a multiplication map $I^{\times n} \rightarrow I$. When this map has an image factorization

$$
I^{\times n} \rightarrow J \hookrightarrow I
$$

such that the associated map $J \rightarrow R$ defines an ideal, we write $I^{n}$ for $J$ and call it the $n^{\text {th }}$ power of $I$. When these exist, the completion of an $n$-pipe ring $R$ at an ideal $I$ is the pro-system of $n$-pipe rings

$$
R_{I}^{\wedge}=\left\{\cdots \rightarrow R / I^{2} \rightarrow R / I\right\}
$$

(Note that because Pro Pro $C$ is naturally equivalent to Pro $C$, this system can again be thought of as an $n$-pipe ring.) The pipe ring $R$ is said to be complete (with respect to the ideal $I$ ) if the natural map $R \rightarrow R_{I}^{\wedge}$ is an isomorphism.

Definition 14 Let $R$ be an $n$-pipe ring. Then for any $x \in \underline{R}$ we have an action map

$$
R=1 \times R \stackrel{x \times \mathrm{id}}{\longrightarrow} R \times R \stackrel{\mu}{\rightarrow} R
$$

From this, we can construct $x^{-1} R$, the localization of $R$ away from $x$, as the ind-object

$$
x^{-1} R=\{R \stackrel{x \cdot-}{\longrightarrow} R \stackrel{x \cdot-}{\longrightarrow} R \stackrel{x \cdot-}{\longrightarrow} \cdots\} .
$$


(Crucially, when $x$ is not invertible in $\underline{R}$, the resulting object will be an $(n+1)-$ pipe ring and not an $n$-pipe ring.) More generally, for any multiplicatively closed subset $T \subset \underline{R}$ we can analogously form $T^{-1} R$. If the natural map $R \rightarrow T^{-1} R$ is an isomorphism, we say that $R$ is local away from $T$.

Remark 15 The operation of localization is idempotent, up to considering an $(n+1)-$ pipe ring as an $(n+2)$-pipe ring.

Proposition 16 Suppose $T \subset \underline{R}$ contains no zero divisors. Then $T^{-1} R$ is fine if $R$ is, and $T^{-1} R$ is cofine if $R$ is.

Proof The maps in the defining ind-system for $T^{-1} R$ induce injections on settheoretic realizations since we are assuming all the elements of $T$ are not zero divisors. On the other hand, inducing up to a pro-system, the cofineness condition is vacuous.

Remark 17 We are deliberately careful to use the notation $x^{-1} R$ to avoid confusing this operation with the construction $R[\alpha] /(x \alpha-1)$. This latter operation is poorly behaved in pipe rings; for instance, the finiteness restriction of Pipes ${ }_{-1}$ can prevent $R[\alpha]$ from existing at all.

It is worth pointing out that it is obviously difficult to get a handle on the closed ideals of a pipe ring in general. In the profinite case of Pipes $_{0}$, there is a comparison with Stone spaces (see Johnstone [11, VI.2.3]) that yields an equivalence

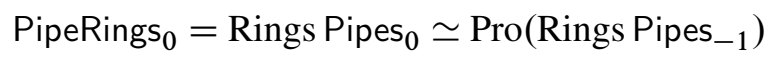

and hence a simple theory of closed ideals, but in general we do not expect any equivalence along these lines.

\subsection{Some formal geometry of pipe rings}

In this section, we generalize basic definitions and facts in formal geometry by introducing the functor of points of a pipe ring. (For a more geometric and less sheaf-theoretic description of $n$-pipe rings, see Appendix B.)

Definition 18 The pipe spectrum (or simply spectrum) of an $n$-pipe ring $R$, denoted $\operatorname{Spp}(R)$, is defined to be the corepresentable functor $\operatorname{Spp}(R)(-)=\operatorname{PipeRings}_{\infty}(R,-)$. Note that this coincides with Spec when restricted to pipe rings of length -1 and with Spf when restricted to pipe rings of length at most 0 . 
Definition 19 We define formal affine $m$-space over the $(n-1)$-pipe ring $R$ to be

$$
\widehat{\mathbb{A}}_{R}^{m}=\operatorname{Spp}\left(R \llbracket x_{1}, \ldots, x_{m} \rrbracket\right) .
$$

Here $R \llbracket x \rrbracket$ denotes the object of PipeRings ${ }_{n}$ given by the pro-system

$$
\cdots \rightarrow R\left\{x^{2}, x^{1}, x^{0}\right\} \rightarrow R\left\{x^{1}, x^{0}\right\} \rightarrow R\left\{x^{0}\right\},
$$

where on underlying $(n-1)$-pipes we define $R\left\{x^{n}, \ldots, x^{0}\right\}=R^{\times(n+1)}$ and endow this with the indicated truncated polynomial ring structure. (Again, the notation is meant to distinguish from the process of forming a polynomial ring and taking quotients, which is not necessarily available.) The multivariate power series ring can be described similarly.

Lemma 20 Maps $\widehat{\mathbb{A}}_{R}^{m} \rightarrow \widehat{\mathbb{A}}_{R}^{k}$ over Spp $R$ are in bijection with $k$-tuples of $m$-variate power series over $\underline{R}$ with all constant terms nilpotent (in the strict sense).

Remark 21 Recall that in classical algebraic geometry, formal schemes arise from completing a scheme at a closed subscheme. This should be thought of as defining a "formal tubular neighborhood" of the closed subscheme. In particular, the new coordinate is privileged by the induced adic topology. This phenomenon is generalized in Definition 19: we should consider the induced map $\operatorname{Spp} R \cong \widehat{\mathbb{A}}_{R}^{0} \rightarrow \widehat{\mathbb{A}}_{R}^{m}$ as the inclusion into a formal neighborhood of dimension $m$.

Definition 22 An $m$-dimensional formal variety over $R$ is a functor

$$
V: \text { PipeRings }_{\infty} \rightarrow \text { Sets }
$$

which is (noncanonically) isomorphic to $\widehat{\mathbb{A}}_{R}^{m}$. A (smooth, 1-dimensional, commutative) formal group over $R$ is a 1-dimensional formal variety $V$ equipped with an abelian group object structure over $\operatorname{Spp} R$. A coordinatized formal group over $R$ is an abelian group object structure on $\widehat{\mathbb{A}}_{R}^{1}$ itself. Finally, the formal group law associated to a coordinatized formal group is given by the representing power series in $\underline{R} \llbracket x, y \rrbracket$ for the multiplication

$$
\widehat{\mathbb{A}}_{R}^{2} \cong \widehat{\mathbb{A}}_{R}^{1} \times_{\text {Spp } R} \widehat{\mathbb{A}}_{R}^{1} \rightarrow \widehat{\mathbb{A}}_{R}^{1} \text {. }
$$

(See Appendix C for a proof of this isomorphism.)

Corollary 23 Over a fixed pipe ring $R$, a coordinatized formal group and a formal group law are equivalent data.

Proof The axioms for a formal group imply that the resulting formal group law must have no constant terms. From here, we appeal to Lemma 20. 
Remark 24 Observe that if $R \rightarrow S$ is a map of $(n-1)$-pipe rings, then the map $\widehat{\mathbb{A}}_{S}^{m} \rightarrow \widehat{\mathbb{A}}_{R}^{m}$ is obtained by pullback along $\operatorname{Spp} S \rightarrow \operatorname{Spp} R$. However, if the pipe rings $R$ and $S$ do not have the same length, taking this pullback will not generally yield a formal affine space over $S$. Hence, it appears that we only obtain the appropriate notion of the "base change" of a formal group by appealing to Corollary 23. (On the other hand, it seems eminently possible that this issue is repaired by passing from formal groups to $p$-divisible groups.)

Classically, formal group laws in positive characteristic are classified according to an invariant called their "height". We define the pipe ring analogue as follows:

Definition 25 Suppose that $R$ is a pipe ring, and select a formal group law $F$ over $R$ with $p$-series given by the formula

$$
[p]_{F}(x)=p x+\sum_{i=2}^{\infty} a_{i} x^{i} \in \underline{R} \llbracket x \rrbracket .
$$

Then, $F$ is said to be of $p$-height $h$ if $R$ is complete with respect to an ideal $I, \underline{I}$ contains $p, \underline{I}$ contains $a_{i}$ for every $i<p^{h}$, and the coefficient $a_{p^{h}}$ is invertible in $R / I$.

To demonstrate that this notion is indeed an isomorphism invariant, we require the following lemma:

Lemma 26 Let $A(x)$ and $A^{\prime}(x)$ be formal power series over the (ordinary) ring $S$ with expansions

$$
A(x)=\sum_{i=1}^{\infty} a_{i} x^{i}, \quad A^{\prime}(x)=\sum_{i=1}^{\infty} a_{i}^{\prime} x^{i},
$$

and let $\varphi(x)$ be a compositionally invertible power series with

$$
A^{\prime}(x)=\varphi^{\circ(-1)}(A(\varphi(x))) .
$$

Suppose that $S$ is complete with respect to some ideal $\mathfrak{a}$. If $A(x)$ has the property that $a_{i} \in \mathfrak{a}$ for $i<n$ and $a_{n} \in S^{\times}$, called property $\boldsymbol{P}_{n}(\mathfrak{a})$, then the property $\boldsymbol{P}_{n}(\mathfrak{a})$ holds for $A^{\prime}(x)$.

Proof Write $\varphi(x)=\sum_{j=1}^{\infty} b_{j} x^{j}$ with $b_{1} \in S^{\times}$. We would like to prove that property $\boldsymbol{P}_{n}(\mathfrak{a})$ holds for the series

$$
A^{\prime}(x)=\varphi^{\circ(-1)}(A(\varphi(x))) .
$$


We begin by proving that property $\boldsymbol{P}_{n}(\mathfrak{a})$ holds for the argument $A(\varphi(x))$, which we expand as

$$
\begin{aligned}
A(\varphi(x))=\sum_{i=1}^{\infty} a_{i} \cdot \varphi(x)^{i} & =\sum_{i=1}^{\infty} a_{i} \cdot\left(\sum_{j=1}^{\infty} b_{j} x^{j}\right)^{i} \\
& =\sum_{i=1}^{\infty} \sum_{j_{1}, \ldots, j_{i}>0} a_{i} b_{j_{1}} \cdots b_{j_{i}} x^{j_{1}+\cdots+j_{i}}=: \sum_{m=1}^{\infty} \beta_{m} x^{m}
\end{aligned}
$$

First, it is clear that $\beta_{m} \in \mathfrak{a}$ for $m<n$, since the coefficients in the penultimate line that sum to give $\beta_{m}$ all contain some factor $a_{i}$ with $i<n$, which by assumption lives in $\mathfrak{a}$. Furthermore, among the coefficients summing to give $\beta_{n}$, we have $a_{n} b_{1}^{n} \in S^{\times}$ when $i=n$ and $b_{j_{1}}=\cdots=b_{j_{i}}=1$, and then all the rest contain some factor $a_{i}$ with $i<n$ and hence live in $\mathfrak{a}$. Thus $\beta_{n} \in S^{\times}$, as the sum of a unit and an element of $\mathfrak{a}$ is again a unit since $S$ is complete with respect to $\mathfrak{a}$. So, $A(\varphi(x))$ does indeed satisfy property $\boldsymbol{P}_{n}(\mathfrak{a})$.

Let us write $\varphi^{\circ(-1)}=\sum_{j=1}^{\infty} c_{j} x^{j}$, which has $c_{1}=b_{1}^{-1} \in S^{\times}$. Consider the expansion

$$
\begin{aligned}
A^{\prime}(x)=\varphi^{\circ(-1)}(A(\varphi(x))) & =\sum_{j=1}^{\infty} c_{j}\left(\sum_{m=1}^{\infty} \beta_{m} x^{m}\right)^{j} \\
& =\sum_{j=1}^{\infty} \sum_{m_{1}, \ldots, m_{j}>0} c_{j} \beta_{m_{1}} \cdots \beta_{m_{j}} x^{m_{1}+\cdots+m_{j}}
\end{aligned}
$$

It is again the case that the coefficients contributing to the terms of order less than $n$ are formed from products of elements in $\mathfrak{a}$, hence are all in $\mathfrak{a}$ themselves. In degree $n$, all but one of the summands contributes a coefficient in $\mathfrak{a}$, where the exception is $c_{1} \beta_{n}$ at $m_{1}=n$. Since this coefficient is a unit, this series also satisfies property $\boldsymbol{P}_{n}(\mathfrak{a})$.

Corollary 27 The $p$-height is independent of the chosen coordinate on a formal group.

Proof Let $G$ and $G^{\prime}$ be two coordinatized formal groups, and let $\varphi$ be an isomorphism between them. Since $R$ is $I$-complete, then $\underline{R}$ is $\underline{I}$-complete. This implies that if an element of $\underline{R}$ becomes invertible in $R / I$, then it is already invertible in $\underline{R}$. Hence we may apply Lemma 26 to the choices $A(x)=[p]_{G}(x)$ and $A^{\prime}(x)=[p]_{G^{\prime}}(x)$. 


\subsection{The Lubin-Tate pipe rings}

For the remainder of the paper, we fix the following data: a finite field $k$ of positive characteristic $p$; a finite, positive integer $h$; a coordinatized formal group $\Gamma$ of finite $p-$ height $h$ over $k$; an integer $N \geq 0$; and a weakly decreasing sequence of nonnegative integers

$$
h=h_{0} \geq h_{1} \geq h_{2} \geq \cdots \geq h_{N} \geq 0 .
$$

As indicated, we will refer to $h_{0}$ simply as $h$, as it plays a privileged role in the theory.

In this section of the paper, we turn to our second desideratum for pipe rings: the instantiation of $\pi_{0} L_{K\left(h^{\prime}\right)} E_{h}$ and its associated localization morphism. Let us recall the basics of the Lubin-Tate theory of deformations of $p$-complete formal group laws of finite height [15]. The space of infinitesimal deformations of the formal group $\Gamma$ of height $h$ chosen above is represented by a formal affine variety $\mathrm{LT}_{h}$ of dimension $h-1$ over the ring $\mathbb{W}_{k}$ of Witt vectors over $k$. This space comes equipped with a nested sequence of relative divisors, identified by a flag of ideals

$$
(0)=I_{0} \leq(p)=I_{1} \leq I_{2} \leq \cdots \leq I_{h} .
$$

This space is not canonically coordinatized; rather, a family of "good" choices of coordinates exist:

Definition 28 Let $\widetilde{\Gamma}$ be a versal (see Artin [4, 3.1]) deformation of the coordinatized formal group $\Gamma$ to $\mathrm{LT}_{h}$. Then, a Lubin-Tate coordinate system on $\mathrm{LT}_{h}$ is a set of coordinate functions $p, u_{1}, \ldots, u_{h-1}$ satisfying the following property for each $t$ :

$$
[p]_{\widetilde{\Gamma}}(x) \equiv u_{t} x^{p^{t}}\left(\bmod \left(p, u_{1}, \ldots, u_{t-1}, x^{p^{t}+1}\right)\right) .
$$

Such coordinate systems always exist. Moreover, the specific choice of versal deformation $\widetilde{\Gamma}$ does not affect whether a given coordinate system is a Lubin-Tate coordinate system. (Indeed, any sequence of elements $u_{t}$ in $I_{t+1} \backslash\left(I_{t} \cup I_{t+1}^{2}\right)$ forms such a system.)

Recall that $\mathrm{LT}_{h} \cong \operatorname{Spf} E_{h}^{0}=\operatorname{Spf} \pi_{0} E_{h}$, where $E_{h}$ is a Morava $E$-theory. Of course, in using this notation we implicitly endow $E_{h}$ with a topology; in fact, it is endowed with a profinite topology, specified by the ideal $I_{h}=\left(p, u_{1}, \ldots, u_{h-1}\right)$, where these generators are taken to form a Lubin-Tate coordinate system. This is the same as considering $\pi_{0} E_{h}$ as a 0 -pipe ring. (Note that this is not quite a formal affine space, since we are deforming away from $p$ in the defining pro-system. This choice aligns with the Lubin-Tate deformation theory of $\Gamma$, which ultimately does not privilege $p$ over the $u_{i}$ for $i \geq 1$.) 
Definition 29 By work of Hovey [7, Lemma 2.3], we have that $\pi_{0} L_{K\left(h_{1}\right)} E_{h} \cong$ $\left(u_{h_{1}}^{-1} \pi_{0} E_{h}\right)_{I_{h_{1}}}^{\wedge}$. More generally, there is the inductive formula

$$
\pi_{0} L_{K\left(h_{n}\right)} \cdots L_{K\left(h_{1}\right)} E_{h} \cong\left(u_{h_{n}}^{-1}\left(\pi_{0} L_{K\left(h_{n-1}\right)} \cdots L_{K\left(h_{1}\right)} E_{h}\right)\right)_{I_{h_{n}}}^{\wedge} .
$$

We inductively endow this with the structure of an $n$-pipe ring: assuming the existence of an $(n-1)$-pipe ring structure on $\pi_{0} L_{K\left(h_{n-1}\right)} \cdots L_{K\left(h_{1}\right)} E_{h}$, we construct the indsystem $u_{h_{n}}^{-1} \pi_{0} L_{K\left(h_{n-1}\right)} \cdots L_{K\left(h_{1}\right)} E_{h}$ as

$$
\left(\pi_{0} L_{K\left(h_{n-1}\right)} \cdots L_{K\left(h_{1}\right)} E_{h} \stackrel{-\cdot u_{h_{n}}}{\longrightarrow} \pi_{0} L_{K\left(h_{n-1}\right)} \cdots L_{K\left(h_{1}\right)} E_{h} \stackrel{-\cdot u_{h_{n}}}{\longrightarrow} \cdots\right),
$$

and from this we construct the pro-system $\left(u_{h_{n}}^{-1}\left(\pi_{0} L_{K\left(h_{n-1}\right)} \cdots L_{K\left(h_{1}\right)} E_{h}\right)\right)_{I_{h_{n}}}^{\wedge}$ as $\left(\cdots \rightarrow\left(u_{h_{n}}^{-1} \pi_{0} L_{K\left(h_{n-1}\right)} \cdots L_{K\left(h_{1}\right)} E_{h}\right) / I_{h_{n}}^{2} \rightarrow\left(u_{h_{n}}^{-1} \pi_{0} L_{K\left(h_{n-1}\right)} \cdots L_{K\left(h_{1}\right)} E_{h}\right) / I_{h_{n}}\right)$.

Lemma 30 The $n$-pipe ring $\pi_{0} L_{K\left(h_{n}\right)} \cdots L_{K\left(h_{1}\right)} E_{h}$ is bifine.

Proof This is immediate from the inductive definition: the quotient maps in the completion systems are all surjective on set-theoretic realizations, and the multiplication maps in the localization systems are all likewise injective.

Proposition 31 The universal formal group law over $\pi_{0} E_{h}$ pulled back to the pipe ring $\pi_{0} L_{K\left(h_{n}\right)} \cdots L_{K\left(h_{1}\right)} E_{h}$ is of $p$-height $h_{n}$.

Proof This follows from the definition of a Lubin-Tate coordinate system, along with the fact that completion and localization have the expected actions on realizations.

As one would hope, the $n$-pipe ring $\pi_{0} L_{K\left(h_{n}\right)} \cdots L_{K\left(h_{1}\right)} E_{h}$ does not depend upon the choice of coordinates, and rather is determined by data stemming from this flag of relative divisors. ${ }^{2}$

Theorem 32 The $n$-pipe ring structure on $\pi_{0} L_{K\left(h_{n}\right)} \cdots L_{K\left(h_{1}\right)} E_{h}$ is independent of choice of Lubin-Tate coordinate system $u_{1}, \ldots, u_{h-1}$ used in its construction.

Proof Let $X_{n-1}^{u}$ denote the pipe ring $\pi_{0} L_{K\left(h_{n-1}\right)} \cdots L_{K\left(h_{1}\right)} E_{h}$ as presented with $u$-coordinates. Suppose that $X_{n-1}^{v}$ is a second such ring, presented in $v$-coordinates, and suppose that the change of coordinates isomorphism $\varphi_{0}: X_{0}^{u} \rightarrow X_{0}^{v}$ has been

${ }^{2}$ Of course, this must be true if we expect to give a formal-geometric interpretation of its functor of points and if the $n$-pipe ring in question indeed comes from a homotopy-theoretic construction which $a$ priori has nothing to do with coordinates. 
shown to extend uniquely to an isomorphism $\varphi_{n-1}: X_{n-1}^{u} \rightarrow X_{n-1}^{v}$. The remainder of the situation is then summarized in the following diagram:

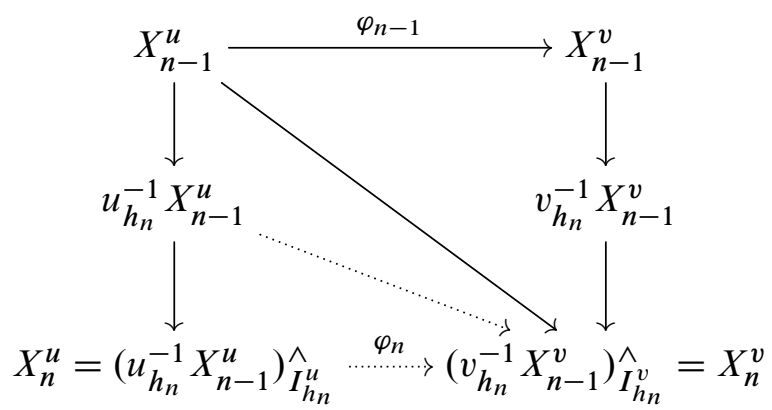

We seek to construct the dashed arrows. (As we will see, in general there is not a map $u_{h_{n}}^{-1} X_{n-1}^{u} \rightarrow v_{h_{n}}^{-1} X_{n-1}^{v}$.) All of the vertical maps are injective on set-theoretic points and all the pipe rings in question are bifine, so the existence of the diagonal dashed arrow is determined by whether $u_{h_{n}}$ is sent to an invertible element in the target. Since

$$
\varphi_{n-1}\left(u_{h_{n}}\right)=v_{h_{n}}+c
$$

for some $c \in I_{h_{n}}^{v}$, then $\varphi_{n-1}\left(h_{n}\right)$ has an inverse in $X_{n}^{v}$ given by

$$
\varphi_{n-1}\left(u_{h_{n}}\right)^{-1}=\frac{1}{v_{h_{n}}+c}=\frac{v_{h_{n}}^{-1}}{1+v_{h_{n}}^{-1} c}=\sum_{j=0}^{\infty}(-1)^{j} c^{j} v_{h_{n}}^{-1-j} .
$$

(Note that the existence of this infinite sum depends on the completeness of $X_{n}^{v}$ against the ideal $I_{h_{n}}^{v}$.) This gives us the diagonal dashed arrow. Finally, we obtain $\varphi_{n}$ from the fact that $\varphi_{n-1}\left(I_{h_{n}}^{u}\right) \subset I_{h_{n}}^{v}$. Switching the two pipe rings and applying this same method produces an inverse to $\varphi_{n}$.

Remark 33 Beilinson and Parshin [5; 8] defined higher local fields to formulate a theory of adèles for higher-dimensional schemes, notably studied in recent years by Osipov $[16 ; 17]$ as well as many others. A necessary input to the construction they consider is a flag of relative divisors, from which they produce a higher-dimensional local field. For instance, the flag of divisors $(0) \leq(x) \leq(x, y)$ in $k[x, y]$ determines the 2-dimensional local field

$$
k((y))((x)):=x^{-1}\left(\left(y^{-1}\left(k[x, y]_{(y)}^{\wedge}\right)\right)_{(x)}^{\wedge}\right),
$$

where $y$ is an element $y \in(x, y) \backslash(x)+(y)^{2}$ and $x$ is an element $x \in(x) \backslash(0)+(x)^{2}$. Hovey's calculation and our presentation above appear to belong to a local, stacky version of their construction. 


\section{A Lubin-Tate theorem}

We remind the reader that we have fixed the following data: a finite field $k$ of positive characteristic $p$; a finite, positive integer $h$; a coordinatized formal group $\Gamma$ of finite $p-$ height $h$ over $k$; an integer $N \geq 0$; and a weakly decreasing sequence of nonnegative integers

$$
h=h_{0} \geq h_{1} \geq h_{2} \geq \cdots \geq h_{N} \geq 0 .
$$

Again, we will often refer to $h_{0}$ simply as $h$, as it plays a privileged role in the theory.

We now describe the moduli problem represented by the pipe spectrum

$$
\operatorname{Spp} \pi_{0} L_{K\left(h_{n}\right)} \cdots L_{K\left(h_{1}\right)} E_{h}
$$

described in the previous section. To do so, we need one further definition:

Definition 34 Write $[N]$ for the category freely generated by a chain of $N$ composable morphisms. We define $N$-staged pipe rings, or StagedRings $N$, to be the full subcategory of Fun([N], PipeRings $\infty)$ of objects $R_{*}$ such that $R_{n}$ is an $n$-pipe ring for each $n \leq N$.

Definition 35 (Staged Lubin-Tate moduli problem) Consider the full subcategory of those $N$-staged pipe rings whose leading term is a complete local ring with residue field $k$, and let $\mathcal{M}=\mathcal{M}_{\Gamma / k}$ be the moduli problem which assigns to such an $N$-staged ring

$$
R_{0} \stackrel{i_{1}}{\rightarrow} R_{1} \stackrel{i_{2}}{\rightarrow} \cdots \stackrel{i_{N}}{\longrightarrow} R_{N}
$$

the following groupoid.

- The objects are given by commuting diagrams of the form

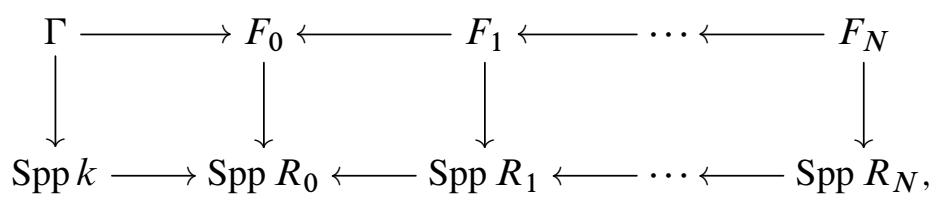

where each $F_{n}$ is a coordinatized formal group over $\operatorname{Spp} R_{n}$ of $p$-height $h_{n}$ whose associated formal group law over $R_{n}$ is obtained by pushing forward that of $F_{n-1}$ along $\underline{i}_{n}$ (so that the arrows in the top row are morphisms of coordinatized formal groups). 
- Morphisms in the groupoid correspond to commuting diagrams of the shape

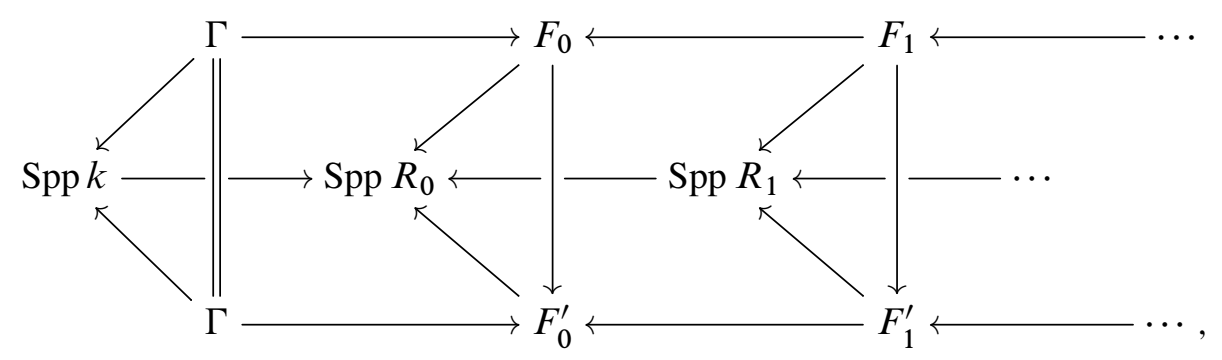

where the first vertical arrow $\Gamma \rightarrow \Gamma$ is an equality and the other vertical arrows are formal group isomorphisms (not necessarily respecting the chosen coordinatizations). Note that the isomorphism $F_{0} \rightarrow F_{0}^{\prime}$ determines the isomorphisms $F_{n} \rightarrow F_{n}^{\prime}$ for all $1 \leq n \leq N$, again by pushing forward along the maps $\underline{i_{n}}$.

We now come to the main theorem:

Theorem 36 (Lubin-Tate theorem for pipe rings) The moduli problem $\mathcal{M}$ is essentially discrete ${ }^{3}$ and is corepresented by the levelwise-bifine $N$-staged pipe ring

$$
\pi_{0} E_{h} \rightarrow \pi_{0} L_{K\left(h_{1}\right)} E_{h} \rightarrow \cdots \rightarrow \pi_{0}\left(L_{K\left(h_{N}\right)} \cdots L_{K\left(h_{1}\right)} E_{h}\right) .
$$

Proof Select a versal deformation $\mathbb{G}_{0}:=\widetilde{\Gamma}$ of the coordinatized formal group $\Gamma$ to $\mathrm{LT}_{h}=\operatorname{Spf} \pi_{0} E_{h}$. Taking $u_{n}$ to be the coefficient of $x^{p^{n}}$ in $[p]_{\mathbb{G}_{0}}(x)$, we obtain an isomorphism $\pi_{0} E_{h} \cong \mathbb{W}_{k} \llbracket u_{1}, \ldots, u_{h-1} \rrbracket$. The elements $p, u_{1}, \ldots, u_{h-1}$ form a Lubin-Tate coordinate system on $\operatorname{Spf} \pi_{0} E_{h}=\mathrm{LT}_{h}$. (Note that these satisfy the required identities on the nose, not just modulo an ideal.) From here on, we refer to the $N$-staged pipe ring in the theorem statement as $X_{*}=\left(X_{0} \rightarrow X_{1} \rightarrow X_{2} \rightarrow \cdots\right)$ and to the pullback of the coordinatized formal group $\mathbb{G}_{0}$ along $\operatorname{Spp} X_{n} \rightarrow \operatorname{Spp} X_{0}$ as $\mathbb{G}_{n}$. These coordinatized formal groups will collectively form our versal object.

At $N=0$, this is the classical Lubin-Tate moduli problem, which is known to be essentially discrete (see Rezk [18, Theorem 4.4]) and representable by $\operatorname{Spp} X_{0}$ [15, Proposition 1.1]. So, suppose that we are given some

$$
R_{*}=\left(R_{0} \stackrel{i_{1}}{\rightarrow} \cdots \stackrel{i_{N}}{\longrightarrow} R_{N}\right) \in \text { StagedRings }_{N}
$$

with $R_{0}$ complete and local with residue field $k$. As a point $\left(F_{0}, \ldots, F_{N}\right) \in \mathcal{M}\left(R_{*}\right)$ is completely determined by the coordinatized formal group $F_{0}$ over $\operatorname{Spp} R_{0}$ (by

\footnotetext{
${ }^{3}$ That is, it is valued in groupoids which are naturally weakly equivalent to sets, or equivalently whose objects admit no nontrivial automorphisms.
} 
virtue of Corollary 23), it follows that $\mathcal{M}$ is a subfunctor of the classical Lubin-Tate moduli problem (pulled back along the forgetful functor to 0 -staged pipe rings, ie to profinite rings), and hence $\mathcal{M}$ is also essentially discrete. This means that the natural transformation

$$
\text { StagedRings }_{N}\left(X_{*},-\right) \rightarrow \mathcal{M}
$$

given on $R_{*}$ by pulling back each $\mathbb{G}_{n}$ along the chosen maps $\operatorname{Spp} R_{n} \rightarrow \operatorname{Spp} X_{n}$ is fully faithful, and so it only remains to check that it is also essentially surjective.

To accomplish this, we will construct a section of the composite natural transformation

$$
\text { StagedRings }_{N}\left(X_{*},-\right) \rightarrow \mathcal{M} \stackrel{\simeq}{\longrightarrow} \pi_{0} \mathcal{M}
$$

More precisely, given a point

$$
\left(F_{0}, \ldots, F_{N}\right) \in \mathcal{M}\left(R_{0} \stackrel{i_{1}}{\rightarrow} \cdots \stackrel{i_{N}}{\longrightarrow} R_{N}\right)
$$

representing an equivalence class in $\pi_{0} \mathcal{M}\left(R_{*}\right)$, we must produce another point in the same path component of $\mathcal{M}\left(R_{*}\right)$ which is in the image of StagedRings ${ }_{N}\left(X_{*}, R_{*}\right)$ under the above natural transformation. Altogether, this amounts to the data of an element $\left\{f_{*}: X_{*} \rightarrow R_{*}\right\} \in$ StagedRings ${ }_{N}\left(X_{*}, R_{*}\right)$ along with commuting isomorphisms of formal groups $\varphi_{n}: F_{n} \rightarrow f_{n}^{*} \mathbb{G}_{n}$ over $\operatorname{Spp} R_{n}$ for $0 \leq n \leq N$. This data fits into the diagram displayed in Figure 1. Noting again that the case $n=0$ is handled by the classical Lubin-Tate moduli problem, we assume that the solid maps have been constructed and that our inductive task is to construct the morphisms $f_{n}$ and $\varphi_{n}$.

Now, let us expand the $p$-series of $F_{n}$ as

$$
[p]_{F_{n}}(x)=p x+\sum_{i=2}^{\infty} a_{i} x^{i} .
$$

By assumption, $F_{n}$ has $p$-height $h_{n}$, and hence $R_{n}$ is complete against the ideal

$$
\mathfrak{a}_{n}:=\left(p, a_{2}, \ldots, a_{p^{h_{n}-1}}\right)
$$

and $a_{p^{h_{n}}}$ is invertible in $\underline{R_{n}}$. In the language of Lemma $26,[p]_{F_{n}}(x)$ has property $\boldsymbol{P}_{n}\left(\mathfrak{a}_{n}\right)$. In light of the isomorphism

$$
F_{n}=i_{n}^{*} F_{n-1} \stackrel{i_{n}^{*} \varphi_{n-1}}{\longrightarrow} i_{n}^{*} f_{n-1}^{*} \mathbb{G}_{n-1},
$$

Lemma 26 implies that the $p$-series of $i_{n}^{*} f_{n-1}^{*} \mathbb{G}_{n-1}$ has property $\boldsymbol{P}_{n}\left(\mathfrak{a}_{n}\right)$ as well. This is exactly what is needed to conclude that the map $i_{n} \circ f_{n-1}$ extends uniquely along the composite

$$
X_{n-1} \rightarrow u_{h_{n}}^{-1} X_{n-1} \rightarrow\left(u_{h_{n}}^{-1} X_{n-1}\right)_{I_{h_{n}}}^{\wedge}=X_{n}
$$




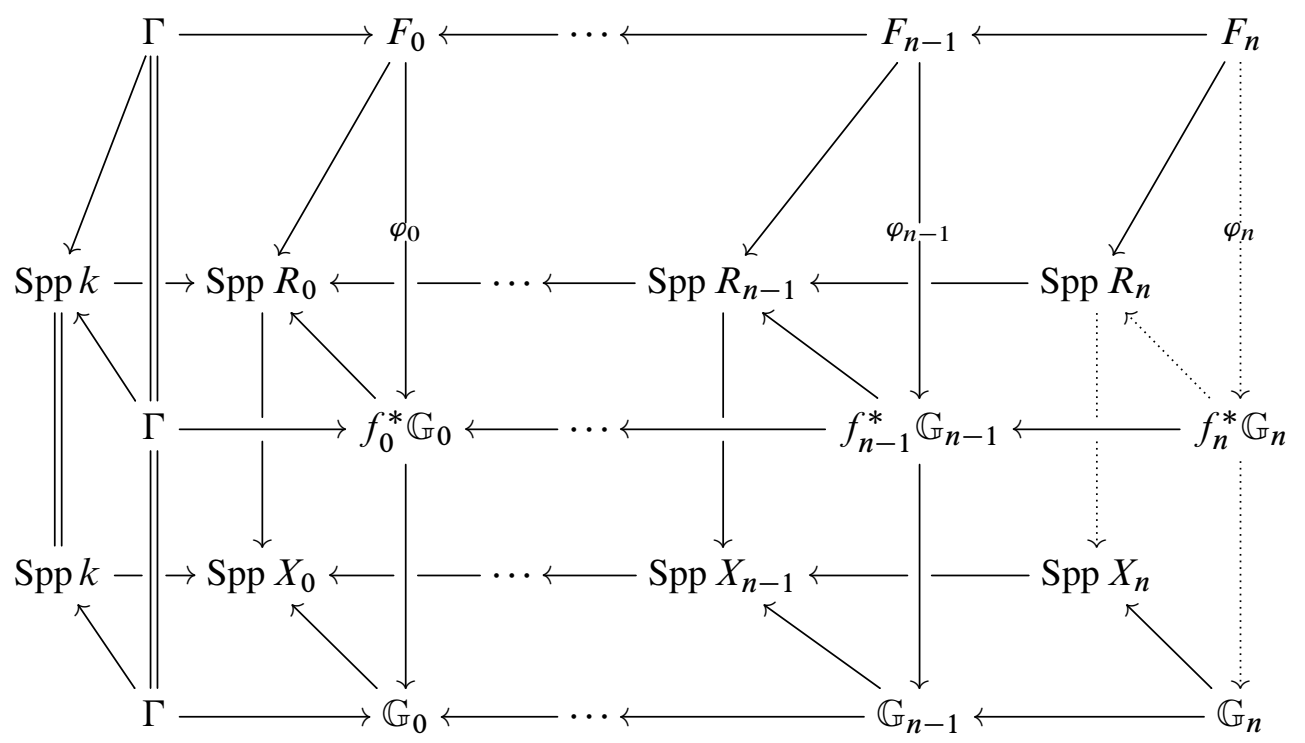

Figure 1: Diagram of the morphisms in play in Theorem 36

We take this extension to be the desired $f_{n}$ and the accompanying isomorphism $\varphi_{n}$ to be

$$
F_{n}=i_{n}^{*} F_{n-1} \stackrel{i_{n}^{*} \varphi_{n-1}}{\longrightarrow} i_{n}^{*} f_{n-1}^{*} \mathbb{G}_{n-1}=f_{n}^{*} \mathbb{G}_{n} .
$$

Remark 37 (Ceci n'est pas une pipe) Our original formulation of this moduli problem used a different source category. Let StagedRings ${ }_{N}$ be defined as the category of commutative squares

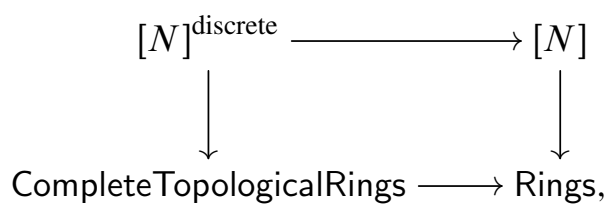

with morphisms commuting natural transformations of the vertical functors. Writing $R^{\prime}$ for the functor $R^{\prime}:[N] \rightarrow$ Rings, this is to say that the morphism of rings $R_{n}^{\prime} \rightarrow R_{n+1}^{\prime}$ within a sequence need not be continuous, but we require that a morphism $R_{n}^{\prime} \rightarrow S_{n}^{\prime}$ across sequences be continuous. Using this category we also define a second moduli problem $\mathcal{M}^{\prime}$, identical in definition to $\mathcal{M}$ except that it uses StagedRings ${ }_{N}^{\prime}$ as a source. For a point $\left(F_{0}^{\prime}, F_{1}^{\prime}, \ldots\right) \in \mathcal{M}^{\prime}\left(R_{*}^{\prime}\right)$, we can also give a Lubin-Tate-type theorem in 
the setting of $\mathcal{M}^{\prime}:$ there is again a unique sequence of compatible maps $f_{n}^{\prime}: \underline{X_{n}} \rightarrow R_{n}^{\prime}$, continuous in the $I_{h_{n}}^{\prime}$-adic topology, expressing $F_{n}^{\prime}$ as the pullback $\left(f_{n}^{\prime}\right)^{*} \overline{\mathbb{G}_{n}}$.

However, this moduli problem "factors" through the moduli problem $\mathcal{M}$ considered here. Note that the set-theoretic realization functor has an obvious lift

$$
\text { StagedRings } \rightarrow \text { StagedRings', }
$$

where we topologize the realizations by, after length 0 , taking our colimits of indsystems and limits of pro-systems in topologized sets - ie in topological spaces rather than simply in sets. (As the forgetful functor Spaces $\rightarrow$ Sets admits both adjoints, these will have the same underlying sets as before.) Given some $R_{*}^{\prime} \in$ StagedRings $_{N}^{\prime}$ with $R_{0}^{\prime}$ profinite, we can construct an $N$-staged pipe ring $R_{*} \in$ StagedRings $_{N}$ by taking $R_{0}=R_{0}^{\prime}$ and $R_{n}=R_{0} \widehat{\otimes}_{X_{0}} X_{n}$ for $n \geq 1$ (defining $\hat{\otimes}$ "levelwise"). This satisfies

$$
\text { StagedRings }_{N}^{\prime}\left(\underline{R_{*}}, R_{*}^{\prime}\right) \stackrel{\cong}{\rightarrow} \operatorname{StagedRings}_{N}^{\prime}\left(\underline{X_{*}}, R_{*}^{\prime}\right) .
$$

It is also worth pointing out that the naive moduli problem $\mathcal{M}^{\prime}$ is often empty: since there is no topological restriction on the maps $R_{n}^{\prime} \rightarrow R_{n+1}^{\prime}$, there is no reason to think that an ideal of definition of $R_{n+1}^{\prime}$ will have as its preimage something contained in the ideal of definition of $R_{n}^{\prime}$. In this situation, no formal group law can have the height property demanded by $\mathcal{M}^{\prime}$. This is all to say that the aggregate category PipeRings $\infty$ directly gives "continuity"-like control over the internal morphisms in these staging sequences, which is not present in the category StagedRings ${ }_{N}$ and whose absence allows for much more pathological behavior there.

\section{Appendix A: $j$-ideals}

We can further refine our definition of closed ideals. Notice that there are $n+1$ naturally occurring inclusions $i_{m}$ of Pipes $n$ into Pipes $n+1$. For instance, in the case of $n=0$, given a profinite set $\left\{X_{\alpha}\right\}_{\alpha}$ one can specify two new pro-ind-profinite sets $i_{0} X$ and $i_{1} X$ using the following pair of formulas:

$$
\left\{\left\{\left(i_{0} X\right)_{\beta}\right\}_{\gamma}\right\}_{\delta}=X_{\delta}, \quad\left\{\left\{\left(i_{1} X\right)_{\beta}\right\}_{\gamma}\right\}_{\delta}=X_{\beta} .
$$

That is, to construct $i_{0} X$ we consider the pro-system $\left\{X_{\alpha}\right\}_{\alpha} \in$ Pipes $_{0}$ as a constant system in Ind Pipes ${ }_{0}$, then consider that as a constant system in Pro(Ind Pipes $\left.{ }_{0}\right)=$ Pipes $_{1}$. On the other hand, to construct $i_{1} X$ we consider each finite set $X_{\alpha} \in$ Pipes $_{-1}$ as a constant system in Pro Pipes $-1=$ Pipes $_{0}$, then consider each of those as a constant system in Ind Pipes ${ }_{0}$, and then finally piece these objects together using the original structure maps of $X$ to get a system in Pro(Ind Pipes 0$)=$ Pipes $_{1}$. The standard 
inclusion Pipes ${ }_{0} \rightarrow$ Pipes $_{1}$ used in the sequential colimit defining Pipes $\infty$ is $i_{0}$. The general pattern is similar, and to capture a coarse part of this phenomenon we make the following definition:

Definition 38 We call a pipe ideal a $j$-ideal if it appears as the kernel of a map to an $j$-pipe ring.

Example 39 The inclusions $i_{m}$ : Pipes $_{0} \rightarrow$ Pipes $_{1}$ can be used to produce examples of strange behavior in pipe rings. For instance, for a finite ring $R \in$ PipeRings $_{-1}$, let $R \llbracket x \rrbracket$ denote the 0 -pipe ring

$$
R \llbracket x \rrbracket:=\left\{\cdots \rightarrow R[x] / x^{3} \rightarrow R[x] / x^{2} \rightarrow R\right\},
$$

and consider the 1-pipe rings

$$
\begin{aligned}
& Q_{0}=i_{0}(R \llbracket x \rrbracket)=\operatorname{const}(R \llbracket x \rrbracket), \\
& Q_{1}=i_{1}(R \llbracket x \rrbracket)=\left\{\cdots \operatorname{const}\left(R[x] / x^{3}\right) \rightarrow \operatorname{const}\left(R[x] / x^{2}\right) \rightarrow \operatorname{const}(R)\right\} .
\end{aligned}
$$

There is a map $Q_{0} \rightarrow Q_{1}$ given by a levelwise quotient $R \llbracket x \rrbracket \rightarrow R[x] / x^{n}$, and while this map realizes to the identity on set-theoretic realizations, no inverse map exists. Instead, its kernel is given by the nonzero pro-ideal $\left\{\left(x^{n}\right)\right\}_{n \geq 1}$, which (given the construction of $Q_{1}$ ) we see is a 1 -ideal. Similar examples can be produced for $n$-pipe rings, $n>1$.

Remark 40 Examples of this flavor also point out an interesting feature of the (co)fine properties defined in Definition 7. The only cofine ideal which realizes to the zero ideal under set-theoretic realization is isomorphic to the zero ideal itself. Hence, no ideal stemming from this sort of construction can ever be cofine. From the perspective of Conjecture 10, this implies that it cannot suffice to simply define the class $W$ of weak equivalences to be those maps which set-theoretically realize to isomorphisms.

Remark 41 This example also illustrates just how wildly set-theoretic realization fails to be conservative. First, one sees that Sets embeds into Pipes 1 via the equivalence Sets $\simeq$ Ind FiniteSets. Then, for any infinite set $X$ with a decreasing filtration of nonempty subsets $X_{\alpha}$ with the property that $\lim _{\alpha} X_{\alpha}=\varnothing$, we can construct an proobject $\tilde{X}$ in Pipes 1 using the inverse system of inclusions. This object has the property that $\underline{\tilde{X}}=\varnothing$, but since there are never any functions from a nonempty set to an empty one, there is no map $\underline{\tilde{X}} \rightarrow \operatorname{const}(\varnothing)$ lifting this isomorphism. 


\section{Appendix B: Portraits}

The point of algebraic geometry in general and schemes in particular is to provide very literally an interface between commutative algebra and geometry. In particular, a scheme comes with a recipe for drawing it, and this is extremely useful for building geometric intuition about the behavior of algebraic objects when it can even partially be carried out. In this section, we produce a construction that provides similar information, and we use it to describe a handful of instructive examples.

Definition 42 We define FilteredSpaces to be the category of sequences

$$
X_{-1} \rightarrow X_{0} \rightarrow X_{1} \rightarrow X_{2} \rightarrow \cdots
$$

of topological spaces with each map the inclusion of a closed subspace. A morphism in this category is a natural transformation of such diagrams (without further restriction).

Definition 43 We define a functor

$$
\square:\left(\text { PipeRings }_{\infty}\right)^{\text {op }} \rightarrow \text { FilteredSpaces, }
$$

called the portrait of a pipe ring. The filtration level $\square(R)_{n}$ is given by the set of those isomorphism classes of closed $m$-ideals of $R$ for $m \leq n$. The topology on $\square(R)_{n}$ is generated by declaring that the closure of a point $I \in \square(R)_{n}$ is the collection of ideals which contain it. Hence, any closed ideal determines a subfiltered space of the portrait $\square(R)$. This construction is functorial in the obvious way.

Remark 44 Our definition of the portrait functor lacks an accompanying locally ringed structure sheaf. We have a definition for the localization of a pipe ring, and so could make an ostensible guess as to a definition of the structure sheaf by mimicking the classical setting, but it does not appear to lead where we would like; see Remark 45. So, we omit it, with some disappointment.

\section{B.1: Example: $k \llbracket x \rrbracket \rightarrow k((x))$}

The basic recipe is to draw a point for each power of each closed prime $n$-ideal, to label the points by the index $n$, and to topologize the space by defining the closure of a point to be the collection of ideals which contain it.

As a first example, let us begin with $k \llbracket x \rrbracket$, where $k$ is a finite field. This 0 -pipe ring has a single closed point corresponding to the kernel of the map $k \llbracket x \rrbracket \rightarrow k$; we represent it by a large dot. Then, there is an ascending sequence of closed nilpotent thickenings of this closed point given by maps

$$
k \llbracket x \rrbracket \rightarrow k[x] / x^{n}
$$


onto nilpotent extensions of $k$. Each of these targets is a finite ring, and so the corresponding ideal is a $(-1)$-ideal. Together, we imagine these as an infinite sequence of smaller dots, topologized as indicated. Finally, there is a single 0 -ideal, corresponding to the identity map and the ideal $(0)$, which we draw as a fuzzy dot at the limit of the smaller ones. As the generic point, its closure is the entire space.

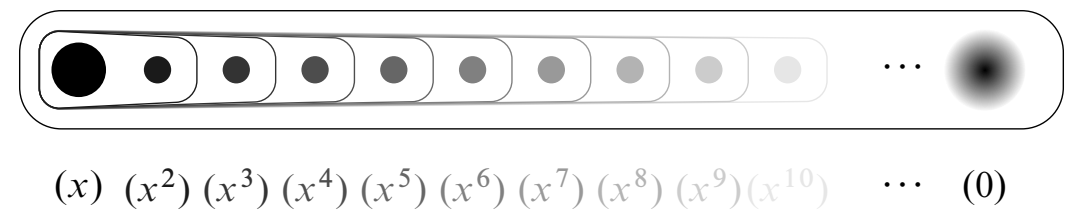

Figure 2: Portrait of $k \llbracket x \rrbracket$ : the solid black dots correspond to $(-1)$-ideals, the fuzzy dot to a 0 -ideal

Given a $k$-algebra $R$, continuous $k$-algebra maps $k \llbracket x \rrbracket \rightarrow R$ pick out elements which can be either nilpotent or merely topologically nilpotent. The difference is detected by whether the corresponding ideal is a $(-1)$ - or 0 -ideal, an observation that will be useful as we now study the ind-profinite ring $k((x))=x^{-1} k \llbracket x \rrbracket$. This ind-object is defined by iterating the multiplication-by- $x$ map, which has a visible action on the ideals: it sends $\left(x^{n}\right)$ to $\left(x^{n+1}\right)$ and (0) to (0). Taking the colimit, we see that $\square(k((x)))$ is supported on the subset (0) of $\square(k \llbracket x \rrbracket)$, and the portrait for $k((x))$ reflects this. We label the point corresponding to (0) with a 1 , to reflect that it is the kernel of a morphism with target in PipeRings 1 . It is also immediately apparent from functoriality of the portrait construction that a morphism of pipe rings $k((x)) \rightarrow R$ cannot send $x$ to a nilpotent element, as the inclusion of the portrait for $k \llbracket x \rrbracket / x^{n}$ into the one for $k \llbracket x \rrbracket$ evidently does not factor through the one for $k((x))$.

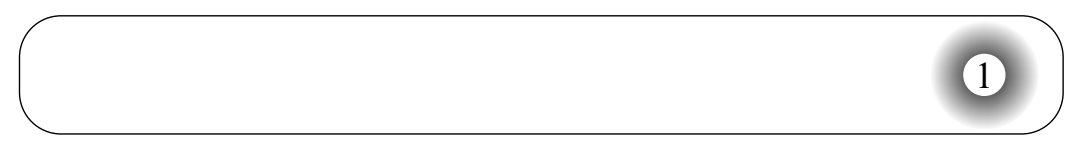

Figure 3: Portrait of $k((x))=x^{-1} k \llbracket x \rrbracket$

Remark 45 Having worked this example, one can explore the obvious definition of the locally ringed structure sheaf that could be attached to this space. The local ring assigned to the point $(x)$ is the ring $k \llbracket x \rrbracket$ itself, but every other point is assigned the field $k((x))$, making it too coarse an invariant to effectively distinguish between the other points we have added. It is unclear how to correct that construction to something more sensitive. 
Remark 46 This same method produces portraits for $\mathbb{Z}_{p}$ and $\mathbb{Q}_{p}$, isomorphic to the ones given above, though it is helpful when working to keep an "arithmetic direction" distinct from the rest.

Remark 47 Throughout, we have taken $k$ to be a finite field, and this is an inescapable feature of our setup. Similar to $k((x))$ in this example, more general local fields can be instantiated as $n$-pipe rings for $n>-1$; see work of Kato [12, Section 1.2].

\section{B.2: Example: $k \llbracket x, y \rrbracket \rightarrow y^{-1} k \llbracket x, y \rrbracket \rightarrow\left(y^{-1} k \llbracket x, y \rrbracket\right)_{(x)}^{\wedge}$}

This is the main example. As before, we proceed in stages, beginning with the 0 -pipe ring $k \llbracket x, y \rrbracket$. This has both closed $(-1)$-ideals and 0 -ideals: examples of $(-1)$-ideals include $(x, y),\left(x^{i}, y^{j}\right)$ for $i, j>0$, and $\left(x^{2}, x y, y^{2}\right)$, whereas examples of 0 -ideals include $(x),\left(x-y^{2}\right),\left(x^{n}\right)$ for $n>0$, and $(x y)$. To analyze the topology, notice first that the closure of an $n$-ideal may only contain $m$-ideals for $m \leq n$. For instance, the 0 -ideal $\left(x^{2}\right)$ is contained in both the 0 -ideal $(x)$ and the $(-1)$-ideal $\left(x^{2}, y\right)$.

To give just an abbreviation of the full portrait, we draw only the points for powers of prime ideals, as in Figure 4. The closed point sits at the bottom left, together with its

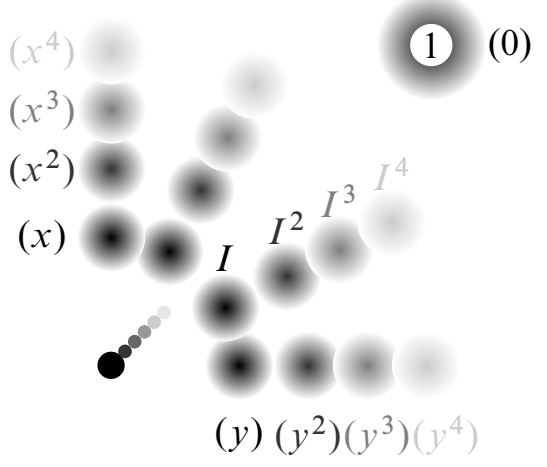

Figure 4: Portrait of $k \llbracket x, y \rrbracket:$ here, $I$ is a closed prime 0 -ideal. The horizontal and vertical axes are labeled by $(y)$ and $(x)$ respectively, as these are the subschemes selected by these ideals.

string of powers. The prime 0 -ideals are also drawn in, with their powers marching away along lines of their own. The ideal $(0)$ is the generic point: it is not contained in the closure of any other point, and its closure is the entire space. This abbreviation is reasonable because the portrait is topologized: we are working in a UFD, so an arbitrary closed ideal will be uniquely characterized by a finite collection of these points. We provide pictures of the closures of the nonprime ideals $\left(y^{3}\right)$ and $\left(x^{3}(x+y)^{2} y\right)$ in Figure 5. 

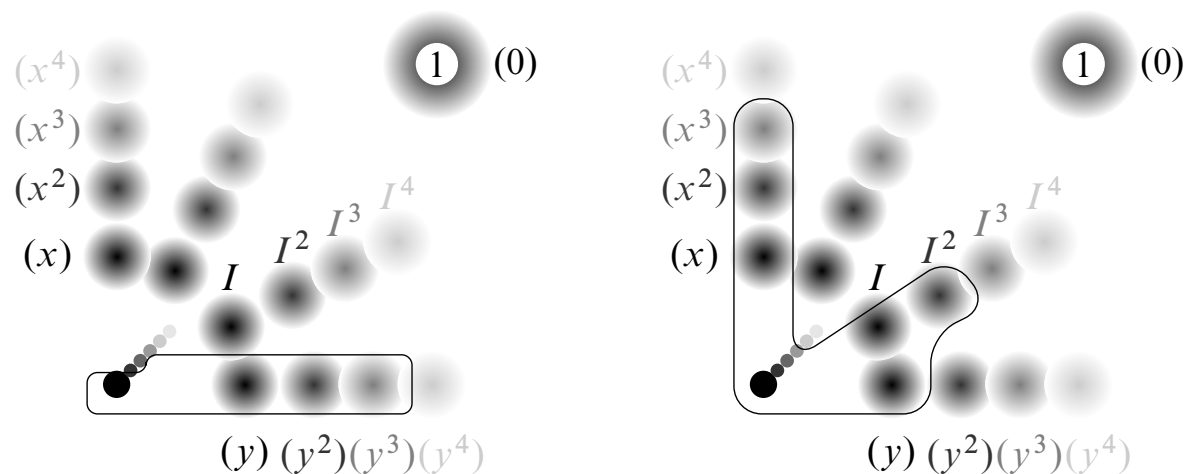

Figure 5: The closures of the ideals $\left(y^{3}\right)$ and $\left(x^{3}(x+y)^{2} y\right)$ in $k \llbracket x, y \rrbracket$ : here, $I$ now represents the specific closed prime 1 -ideal $(x+y)$.

We now seek to understand the intermediate ring $y^{-1} k \llbracket x, y \rrbracket$. Just as before, multiplication by $y$ sends a closed set corresponding to $I$ to the closed set corresponding to $y I$. To produce a portrait of this pipe ring, we identify the closed sets associated to $I$ and $J$ when

$$
\bigcup_{k} \overline{y^{k} I}=\bigcup_{k} \overline{y^{k} J}
$$

In terms of the abbreviated portrait, this action admits a simple description: a closed set in the original portrait is sent to its union with $\left\{(y),\left(y^{2}\right), \ldots\right\}$.

However, there are an awful lot of ideals (ie closed sets) in $y^{-1} k \llbracket x, y \rrbracket$. This uncomfortable fact is completely done away with by completing against the ideal $(x)$, producing the ring $\left(y^{-1} k \llbracket x, y \rrbracket\right)_{(x)}^{\wedge}$. The Weierstrass preparation theorem tells us that any element $f(x, y)$ of this ring takes the form

$$
f(x, y)=x^{n} \cdot g(x, y),
$$

where $g(x, y)$ is a unit (or equivalently, considered as a power series in $x$ its constant coefficient is a unit). It is instructive to note that this completion allows for power series that extend infinitely in both directions in $y$, provided that the coefficients of the negative powers of $y$ lie in increasing powers of the ideal $(x)$. This reflects the fact that there is an isomorphism (of pipe rings)

$$
\left(y^{-1} k \llbracket x, y \rrbracket\right)_{(x)}^{\wedge} \cong k((y)) \llbracket x \rrbracket .
$$

Pictorially, this completion also has a very simple description: two closed sets are identified if they have the same intersection with the set $\left\{(x),\left(x^{2}\right), \ldots\right\}$. For example, 
the closed sets defined by the ideals $\left(x^{2}(x+y)^{3} y^{2}\right)$ and $\left(x^{2}\right)$ become identified because $(x+y)^{3} y^{2}$ is now a unit.

(2) (0)

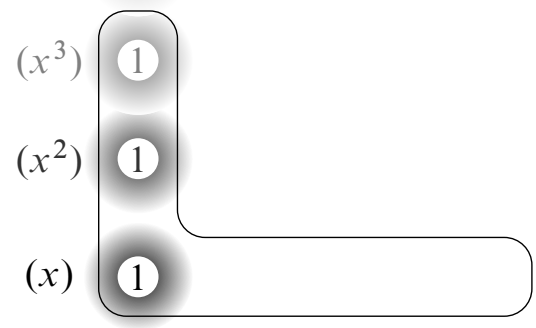

Figure 6: Portrait of $\left(y^{-1} k \llbracket x, y \rrbracket\right)_{(x)}^{\wedge}$, with the closure of $\left(x^{3}\right)$ indicated: this closed set has been drawn with the distention to suggest that it has been inherited from the previous stage $y^{-1} k \llbracket x, y \rrbracket$ of the construction.

\section{Appendix C: Complete elements and products of formal affine space}

It turns out to be slightly subtle to prove that finite products of formal affine spaces are again formal affine spaces. One would hope to prove such a statement using some argument involving Yoneda's lemma. However, given some $R \in$ PipeRings $_{n-1}$, on the one hand it is clear that $R \llbracket x_{1}, \ldots, x_{n} \rrbracket$ satisfies some universal property in PipeRings ${ }_{n}$, but it is much less clear that this persists in the larger category PipeRings $\infty$. To deduce the result, we must extend the theory developed in Section 2.2.

We begin with the following generalization of Definition 19.

Definition 48 Let $R$ be an (n-1)-pipe ring, and choose any compactly supported function $\boldsymbol{m}: \mathbb{Z}_{\geq n} \rightarrow \mathbb{Z}_{\geq 0}$. For convenience, we write $\boldsymbol{m}_{i}=\boldsymbol{m}(i)$, and choose $k$ such that $\boldsymbol{m}_{i}=0$ for $i>k$. Then, we define formal affine $\boldsymbol{m}$-space over $R$ to be the pipe spectrum of the $k$-pipe ring

$R \llbracket \boldsymbol{x}_{\boldsymbol{m}} \rrbracket=\left(\cdots\left(\left(R \llbracket x_{1, n}, \ldots, x_{\boldsymbol{m}_{n}, n} \rrbracket\right) \llbracket x_{1, n+1}, \ldots, x_{\boldsymbol{m}_{n+1}, n+1} \rrbracket\right) \cdots\right) \llbracket x_{1, k}, \ldots, x_{\boldsymbol{m}_{k}, k} \rrbracket$.

That is, at each length $i$, beginning at $i=n$ and ending at $i=k$, we adjoin $\boldsymbol{m}_{i}$ new power series variables. In other words, we are forming formal affine $\boldsymbol{m}_{k}$-space over ... over formal affine $\boldsymbol{m}_{n}$-space over $R$. This all reduces to $\widehat{\mathbb{A}}_{R}^{m}$ if we take $\boldsymbol{m}_{n}=m$ and $\boldsymbol{m}_{i}=0$ for $i>n$. 
This allows us to define "complete elements" of pipe rings under a given pipe ring.

Definition 49 Let $R$ be an (n-1)-pipe ring, let $R \rightarrow S$ be a map of pipe rings, and let $\boldsymbol{m}: \mathbb{Z}_{\geq n} \rightarrow \mathbb{Z}_{\geq 0}$ be as above. We define an $\boldsymbol{m}$-tuple of complete elements of $S$ as an $R$-algebra to be a map $R \llbracket x_{\boldsymbol{m}} \rrbracket \rightarrow S$ extending the structure map $R \rightarrow S$.

We now arrive at the desired universal characterization of formal affine space.

Proposition 50 Let $R$ be an $(n-1)$-pipe ring. Then for any pipe ring $S$, maps $R \llbracket x_{1}, \ldots, x_{m} \rrbracket \rightarrow S$ are in bijection with pairs of a map $R \rightarrow S$ in PipeRings $\infty$ and an $\boldsymbol{m}$-tuple of complete elements of $S$, where $\boldsymbol{m}_{n}=m$ and $\boldsymbol{m}_{i}=0$ for $i \neq n$.

Corollary 51 Let $R$ be an $(n-1)$-pipe ring. Then

$$
\widehat{\mathbb{A}}_{R}^{m} \times_{\operatorname{Spp} R} \widehat{\mathbb{A}}_{R}^{k} \cong \widehat{\mathbb{A}}_{R}^{m+k}
$$

for any $m, k \geq 0$.

Remark 52 The category of pipe rings does not admit an initial object, but one could nevertheless make an absolute version of Definition 49 by asking for multiplicative maps to $(S, \cdot)$ from "the free abelian semigroup with zero in Pipes $_{\infty}$ containing an $\boldsymbol{m}$-tuple of complete elements". We will leave this to the reader's imagination, where it is sure to be more vivid.

\section{References}

[1] M Ando, C P French, N Ganter, The Jacobi orientation and the two-variable elliptic genus, Algebr. Geom. Topol. 8 (2008) 493-539 MR2443236

[2] M Ando, J Morava, A renormalized Riemann-Roch formula and the Thom isomorphism for the free loop space, from: "Topology, geometry, and algebra: Interactions and new directions", (A Adem, G Carlsson, R Cohen, editors), Contemp. Math. 279, Amer. Math. Soc. (2001) 11-36 MR1850739

[3] M Ando, J Morava, H Sadofsky, Completions of $\mathbb{Z} /(p)$-Tate cohomology of periodic spectra, Geom. Topol. 2 (1998) 145-174 MR1638030

[4] M Artin, Versal deformations and algebraic stacks, Invent. Math. 27 (1974) 165-189 MR0399094

[5] A A Berlinson, Residues and adèles, Funktsional. Anal. i Prilozhen. 14 (1980) 44-45 MR565095 In Russian; translated in Functional Anal. Appl. 14 (1980) 34-35

[6] J P C Greenlees, N P Strickland, Varieties and local cohomology for chromatic group cohomology rings, Topology 38 (1999) 1093-1139 MR1688422 
[7] M Hovey, Bousfield localization functors and Hopkins' chromatic splitting conjecture, from: "The Čech centennial”, (M Cenkl, H Miller, editors), Contemp. Math. 181, Amer. Math. Soc. (1995) 225-250 MR1320994

[8] A Huber, On the Parshin-Bellinson adèles for schemes, Abh. Math. Sem. Univ. Hamburg 61 (1991) 249-273 MR1138291

[9] D C Isaksen, A model structure on the category of prosimplicial sets, Trans. Amer. Math. Soc. 353 (2001) 2805-2841 MR1828474

[10] D C Isaksen, Calculating limits and colimits in procategories, Fund. Math. 175 (2002) 175-194 MR1969635

[11] P T Johnstone, Stone spaces, Cambridge Studies in Advanced Math. 3, Cambridge Univ. Press (1982) MR698074

[12] K Kato, Existence theorem for higher local fields, from: "Invitation to higher local fields”, (I Fesenko, M Kurihara, editors), Geom. Topol. Monogr. 3 (2000) 165-195 MR1804933

[13] K Kato, S Saito, Global class field theory of arithmetic schemes, from: "Applications of algebraic $K$-theory to algebraic geometry and number theory, Part I, II", (S J Bloch, R K Dennis, E M Friedlander, M R Stein, editors), Contemp. Math. 55, Amer. Math. Soc. (1986) 255-331 MR862639

[14] T Lawson, N Naumann, Commutativity conditions for truncated Brown-Peterson spectra of height 2, J. Topol. 5 (2012) 137-168 MR2897051

[15] J Lubin, J Tate, Formal moduli for one-parameter formal Lie groups, Bull. Soc. Math. France 94 (1966) 49-59 MR0238854

[16] D V Osipov, The Krichever correspondence for algebraic varieties, Izv. Ross. Akad. Nauk Ser. Mat. 65 (2001) 91-128 MR1874355

[17] D V Osipov, $n$-dimensional local fields and adèles on $n$-dimensional schemes, from: "Surveys in contemporary mathematics", (N Young, Y Choi, editors), London Math. Soc. Lecture Note Ser. 347, Cambridge Univ. Press (2008) 131-164 MR2388492

[18] C Rezk, Notes on the Hopkins-Miller theorem, from: "Homotopy theory via algebraic geometry and group representations”, (M Mahowald, S Priddy, editors), Contemp. Math. 220, Amer. Math. Soc. (1998) 313-366 MR1642902

[19] S Saito, Arithmetic on two-dimensional local rings, Invent. Math. 85 (1986) 379-414 MR846934

[20] S Saito, Class field theory for two-dimensional local rings, from: "Galois representations and arithmetic algebraic geometry”, (Y Ihara, editor), Adv. Stud. Pure Math. 12, North-Holland (1987) 343-373 MR948252

[21] T M Schlank, N Stapleton, A transchromatic proof of Strickland's theorem arXiv: 1404.0717 
[22] N Stapleton, Transchromatic generalized character maps, Algebr. Geom. Topol. 13 (2013) 171-203 MR3031640

[23] N Stapleton, Transchromatic twisted character maps, J. Homotopy Relat. Struct. 10 (2015) 29-61 MR3313634

[24] T Torii, On degeneration of one-dimensional formal group laws and applications to stable homotopy theory, Amer. J. Math. 125 (2003) 1037-1077 MR2004428

[25] T Torii, Milnor operations and the generalized Chern character, from: "Proceedings of the Nishida Fest", (M Ando, N Minami, J Morava, W S Wilson, editors), Geom. Topol. Monogr. 10 (2007) 383-421 MR2402795

[26] T Torii, Comparison of Morava E-theories, Math. Zeitschrift 266 (2010) 933-951 MR2729298

[27] T Torii, HKR characters, p-divisible groups and the generalized Chern character, Trans. Amer. Math. Soc. 362 (2010) 6159-6181 MR2661512

[28] T Torii, $K(n)$-localization of the $K(n+1)$-local $E_{n+1}$-Adams spectral sequences, Pacific J. Math. 250 (2011) 439-471 MR2794609

AMG, EP: Department of Mathematics, University of California Berkeley 970 Evans Hall \#3840, Berkeley, CA 94720-3840, USA

NS: Department of Mathematics, Massachusetts Institute of Technology 77 Massachusetts Avenue E18-369, Cambridge, MA 02139-4307, USA aaron@math.berkeley.edu, ericp@math.berkeley.edu, nstapleton@math.mit.edu http://math.berkeley.edu/ aaron/, http://math.berkeley.edu/ ericp/, http://math.mit.edu/ nstapleton/

Received: 8 May 2014 Revised: 18 September 2014 